\title{
Research Paper \\ The Effects of Sodium Hydrosulfide on Motor Learning in a Rat Model of Parkinson's Disease
}

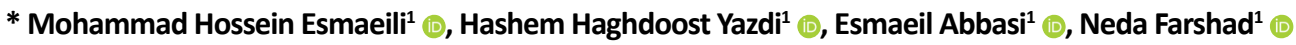

Keywords:

Hydrogen Sulfide, 6-Hydroxydopamine, Rotarod test, Medial Forebrain Bundle (MFB), Parkinson's Disease

\section{ABSTRACT}

Background Hydrogen Sulfide $\left(\mathrm{H}_{2} \mathrm{~S}\right)$, a novel endogenous gasotransmitter, plays an important role in neuromodulation and memory performance and also protects neurons against neurotoxin-induced neurodegeneration.

Objective This study aimed to investigate the potential neuroprotective effects of Sodium Hydrosulfide (NaHS), on motor learning in a unilateral 6-Hydroxydopamine (6-OHDA) rat model of Parkinson's Disease. Methods Male Wistar rats were subjected to unilateral injection of 6-OHDA (15 $\mu \mathrm{g}$ ) into the Medial Forebrain Bundle (MFB) and then treated with NaHS for 25 days. Animals were divided into control, sham, sham plus NaHS, Parkinson (6-OHDA), Parkinson plus vehicle (saline), and Parkinson plus NaHS (2.8 and $5.6 \mathrm{mg} /$ $\mathrm{kg}$, IP) groups, ( $\mathrm{N}=8)$. One-way ANOVA followed by turkey's test was applied for statistical analyses of the data.

Findings The riding time in fixed and accelerating speed rotarod were significantly decreased in Parkinson rats (6-OHDA group) compared to controls in all training days $(\mathrm{P}<0.001)$. Treatment with NaHS (2.8 and 5.6 $\mathrm{mg} / \mathrm{kg} / \mathrm{d}$ ) reversed these decreases in a dose-dependent manner, so no significant differences were found in these parameters between the control and Parkinson plus NaHS groups during the accelerating speed rotarod test.

Conclusion In the Parkinson rats, NaHS administration enhanced and improved the endurance time in the rotarod test. These results demonstrate that NaHS treatment enhances rat motor balance and coordination and suggest treatment with NaHS attenuates motor impairments in the Parkinson rats.

\section{Extended Abstract}

\section{Introduction}

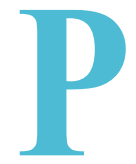

arkinson Disease (PD) is a progressive neurodegenerative disorder characterized by a progressive loss of Substantia Nigra pars compacta $(\mathrm{SNc})$ dopaminergic neurons, which leads to motor symptoms of ance [1]. PD is a neurodegenerative condition in which dementia may be observed even in the early stage of the disease together with mild motor disabilities that follows midbrain dopaminergic neuron loss [2].

The prevalence of cognitive dysfunction is high with reports indicating that this non-motor symptom negatively affects the quality of life of about $60 \%$ of patients with PD and cause morbidity and mortality in $36 \%$ of patients

bradykinesia, rigidity, resting tremor, and postural imbal-

\section{* Corresponding Author:}

Mohammad Hossein Esmaeili, PhD.

Address: Department of Physiology and Biophysics, School of Medicine, Qazvin University of Medical Sciences, Qazvin, Iran.

Tel: +98 (281) 3336002-5

E-Mail: esmail66@yahoo.com 
at the early onset of the disease [3]. Therefore, the patients suffering from PD, appear to be particularly susceptible to develop cognitive impairments 4-6 times more than what happens with normal aging [4].

Both structural and functional changes of the hippocampus which are involved in cognitive processes such as learning and memory have been observed in PD patients [5, 6]. Several studies have also shown that the reduction of hippocampal volume was accompanied by cognitive deficits in PD patients [7]. Numerous studies have suggested that neuroinflammation and oxidative stress play major roles in the pathogenesis of nigral dopaminergic cell death in PD [8].

6-Hydroxydopamine (6-OHDA) was the first dopaminergic neurotoxin used experimentally to induce PD [9]. The neuronal damage induced by 6-OHDA is mainly due to the great oxidative stress caused by the toxin. For this reason, unilateral injection of 6-OHDA is a good model for studying oxidative stress in the pathogenesis of PD. To date, three main gasotransmitters have been identified: Nitric Oxide (NO), Carbon monoxide (CO), and Hydrogen Sulfide $\left(\mathrm{H}_{2} \mathrm{~S}\right)$. It has been discovered that $\mathrm{H}_{2} \mathrm{~S}$ plays multiple functional roles in the body from cardiovascular tone regulation to neuromodulation.

In recent years, it has been demonstrated that $\mathrm{H}_{2} \mathrm{~S}$ as a cytoprotectant, significantly improves spatial learning and memory impairment induced by $\mathrm{A} \beta$ and exerts anti-inflammatory, anti-apoptotic, and antioxidant effects [10]. However, the possible role of $\mathrm{H}_{2} \mathrm{~S}$ as an anti-antioxidant agent in cognitive dysfunction in a rat model of PD has not yet been fully elucidated. Therefore, the present study was designed to investigate the protective effects of $\mathrm{H}_{2} \mathrm{~S}$ against 6-OHDA-induced motor learning impairment in a unilateral 6-OHDA rat model of PD.

\section{Methods and Materials}

Animals were first anesthetized with Intraperitoneal (IP) injection of ketamine $(100 \mathrm{mg} / \mathrm{kg})$ and xylazine $(10 \mathrm{mg} /$ $\mathrm{kg}$ ) and were then placed in a stereotactic apparatus. Burr holes were drilled in the right side of the skull and $4 \mu \mathrm{g}$ of 6-OHDA mixed in $2 \mu \mathrm{L}$ of $0.2 \%$ ascorbic acid with $0.9 \%$ normal saline was injected into the ascending mesostriatal pathway using stereotactic positioning ( $4.4 \mathrm{~mm}$ posterior to the bregma, $1.2 \mathrm{~mm}$ lateral to the midline, and $7.8 \mathrm{~mm}$ below the dura) near the MFB at a rate of $0.2 \mu \mathrm{L}$.

Administration of normal saline vehicle with the same volume was performed as sham surgery. Then the animals were divided into seven groups; including control, sham, sham plus NaHS (5.6 mg/kg), Parkinson (6-OHDA), Parkinson plus vehicle (saline), and Parkinson plus NaHS (2.8 and 5.6 $\mathrm{mg} / \mathrm{kg}$ ). Animals in the sham plus NaHS and 6-OHDA plus $\mathrm{NaHS}$ groups received daily intraperitoneal administration of NaHS (Sigma-USA) post-operation for 25 days. NaHS was dissolved in distilled water. Behavioral tests were performed $24 \mathrm{~h}$ after the last injection. Motor coordination was assessed using an automated rotating rod $[11,12]$. One-way ANOVA was applied for statistical analyses of the data. The level of significance was less than 0.05 .

\section{Results}

The overall results showed no statistically significant differences in different parameters between the sham, control, and sham plus NaHS groups. Furthermore, no statistically significant difference was found between the 6-OHDA and 6-OHDA plus vehicle (saline) groups with respect to different parameters. Data are not shown. Therefore, these data are not depicted graphically and the data obtained from the 6-OHDA plus saline (6-OHDA group) and 6-OHDA plus

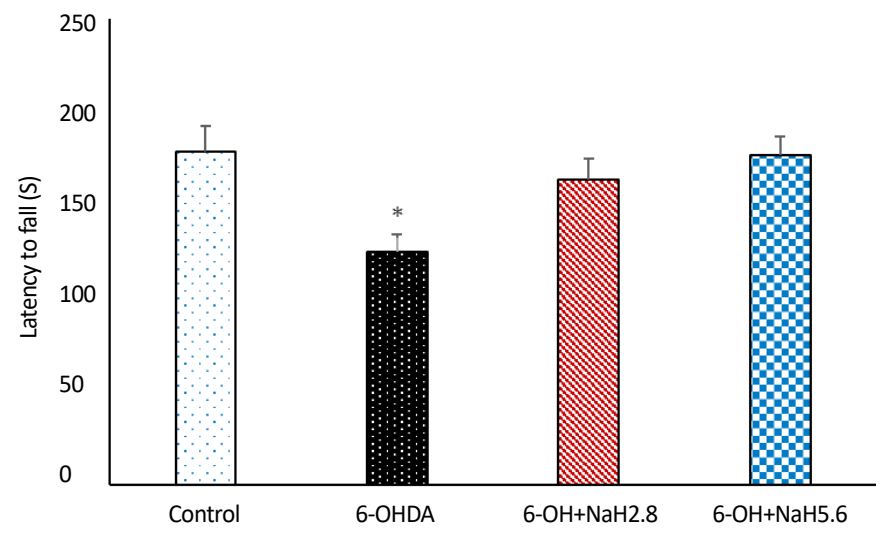

Figure 1. Comparison of latency to fall in control and experimental groups 
NaHS (2.8 and $5.6 \mathrm{mg} / \mathrm{kg}$ ) groups were compared with each other and with the control group.

The riding time in fixed and accelerating speed rotarod significantly decreased in Parkinson rats (6-OHDA group) compared to controls, in all of the training days. Treatment with NaHS (2.8 and $5.6 \mathrm{mg} / \mathrm{kg} / \mathrm{d}$ ) reversed these decreases in a dose-dependent manner, so no significant differences were found between the control and Parkinson plus NaHS groups in these parameters during the fixed and accelerating speed rotarod test (Figure 1).

Our results showed that microinjection of 6-OHDA into the MFB depressed motor performances of animals on the rotarod and remarkably decreased the time that animals balanced steadily on the rotarod, whereas administration of NaHS (2.8 and $5.8 \mathrm{mg} / \mathrm{kg}$ ) reversed the inhibitory effects of 6-OHDA on rotarod and considerably increased the animals' endurance time on rotarod. These results demonstrate that NaHS enhances rat motor balance and coordination in a unilateral 6-OHDA rat model of PD.

\section{Conclusion}

In the Parkinson rat group, NaHS administration enhanced and improved the endurance time in the rotarod test. These results demonstrate that NaHS treatment enhances rat motor balance and coordination and suggest that treatment with NaHS attenuates motor impairments in the Parkinson rat group.

\section{Ethical Considerations}

\section{Compliance with ethical guidelines}

This research was approved by the Ethics Committee of the Qazvin University of Medical Sciences (code: IR.QUMS.REC.1396.476).

\section{Funding}

The present paper was extracted from the MSc. thesis of Neda Farshad, in Department of Physiology and Biophysics, School of Medicine, Qazvin University of Medical Sciences.

\section{Authors' contributions}

Conception, design and revising the article critically for important intellectual content: Mohammad Hossein Esmaeili; acquisition, Analysis and interpretation of data: Mohammad Hossein Esmaeili, Esmaeil Abbasi, Neda Farshad; and Drafting the article: Mohammad Hossein Esmaeili, Hashem Haghdoost Yazdi.

\section{Conflict of interest}

The authors declared no conflict of interest.

\section{Acknowledgments}

The authors would like to thank the Department of Physiology, School of Medical Sciences, Qazvin University of Medical Sciences. 


\title{
بررسى اثرات هيدروسولفيد سديم بر يادكيرى حركتى در مدل موش صحرايى ياركينسونى
}

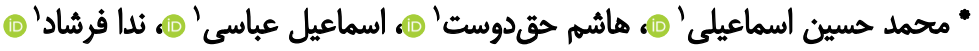 \\ 1- كروه فيزيولوزى و فيزيك يزشكى، دانشكده يزشكى، دائشكاه علوم يزشكى قزوين، قزوين، ايران.
}

\begin{abstract}
דيكيد

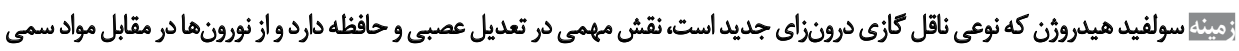
تخريبكنئلهمحافظت مئى كئل

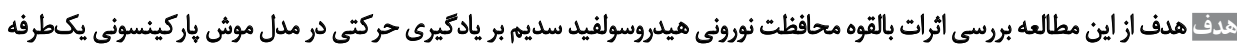

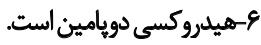

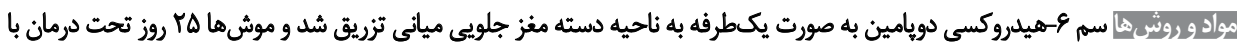

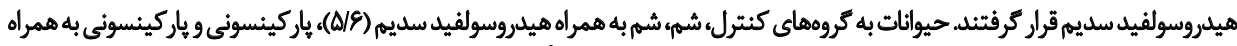

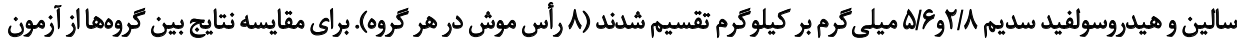

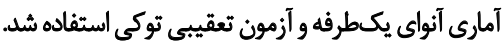

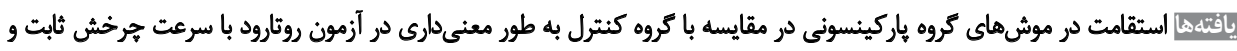

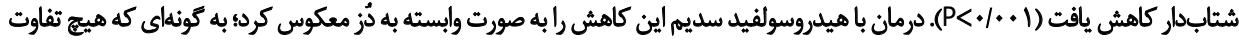

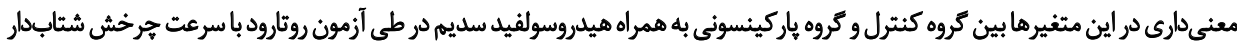
مئاهدهنشئ.

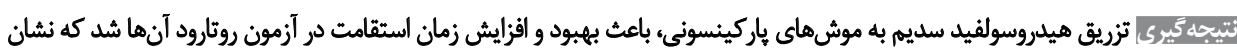

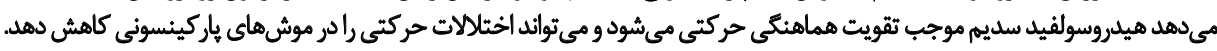

تاريخ دريافت: 9 خرداد Vو IV

تاريخ بذيبش: 4 شهريور

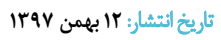

كليدوإوها:

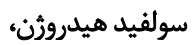

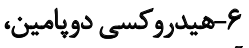

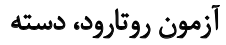

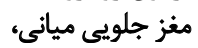
بيمارى ياركينسون

محصولات فرعى توليد مى موند كه باعث آسيب به ميتوكندرى

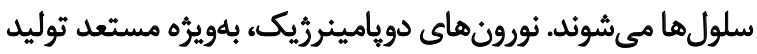

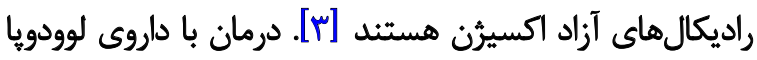

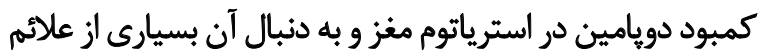

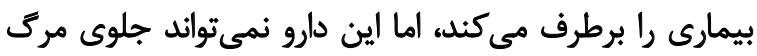

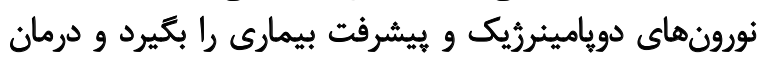

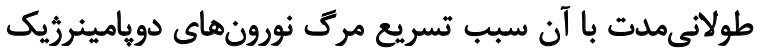

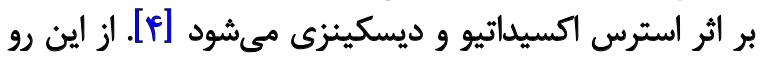

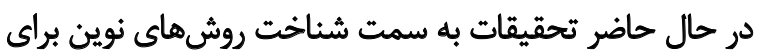

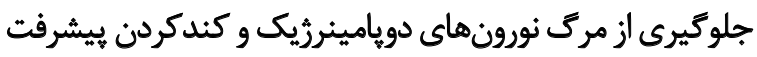
بيمارى محرود. سولفيد هيدرورن نوعى مولكول سيكَالينَ درونزاست و

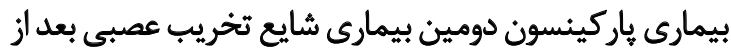

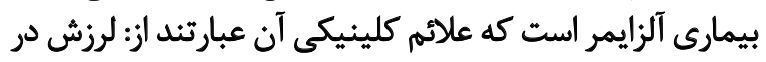

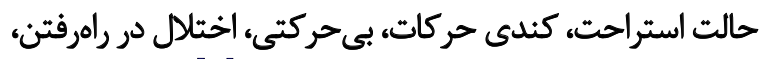

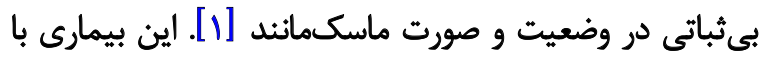

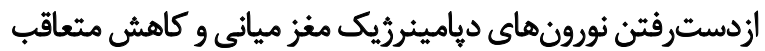

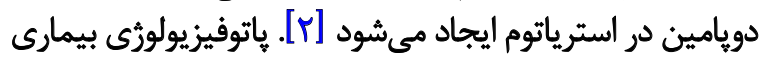

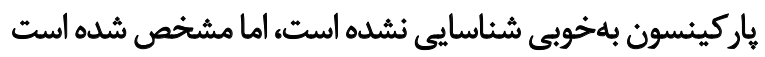

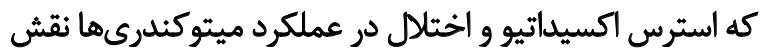

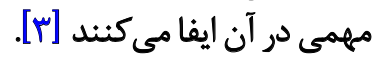

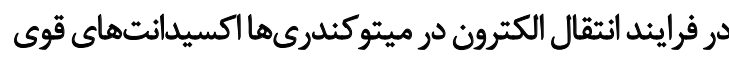

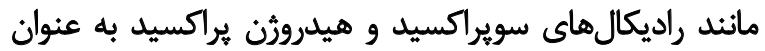




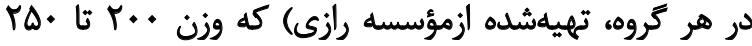

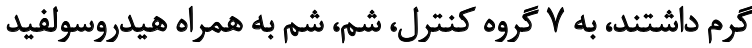

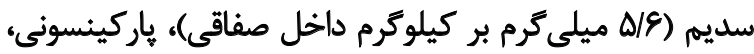

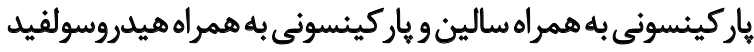

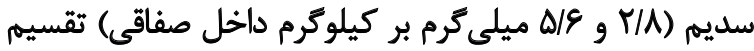

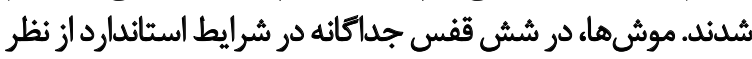

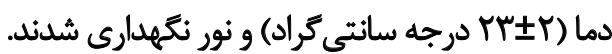
در مدت آزمايش، موشها آب و غذاى طبيعى خود را آزادانه

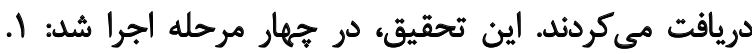

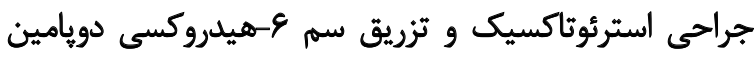

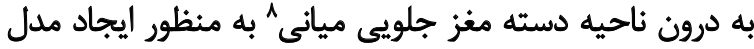

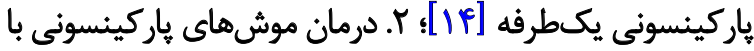

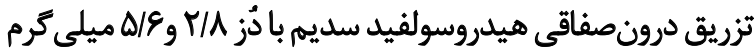

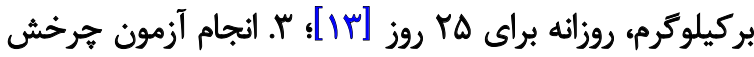

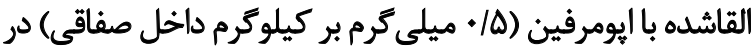

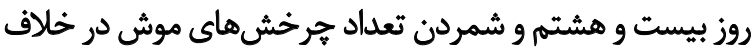

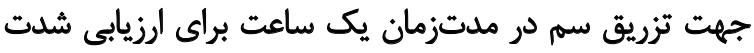

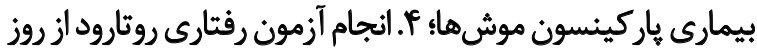

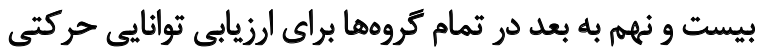

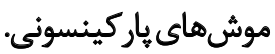

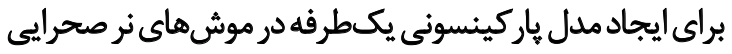

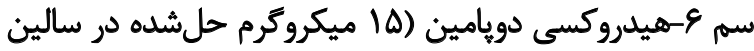

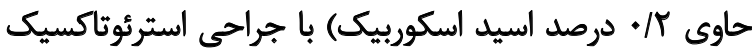

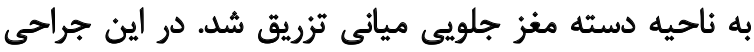

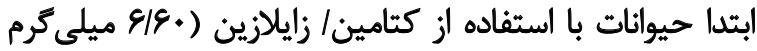

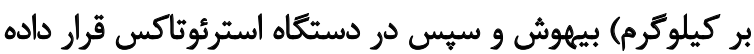

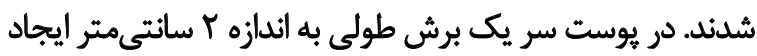

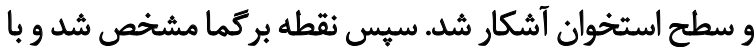

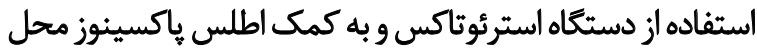

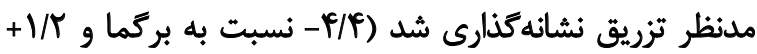
نسبت به خط وسط و V/A نسبت به سخت شامه).

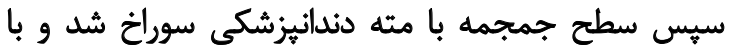

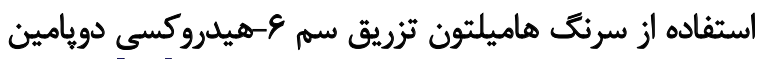

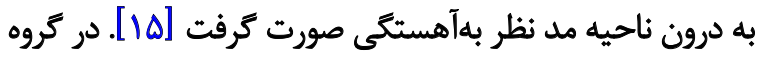

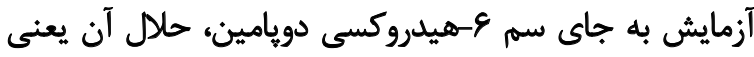

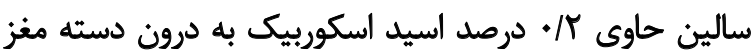
جلويى ميانى تزريق شد.

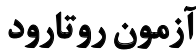

به كمك اين آزمون هماهنكى حركات، حفظ تعادل و مهمتر

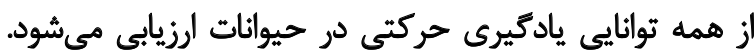

\section{Medial Forebrain Bundle (MFB)}

بس از منوكسيدكربن و نيتروكسايد، به عنوان ناقل كازى سوم

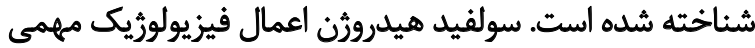

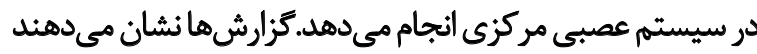

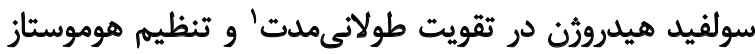

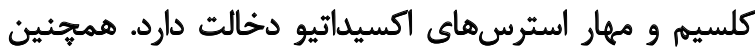

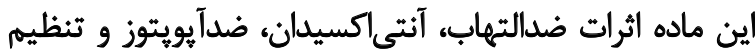

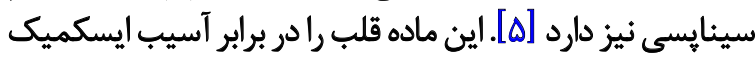

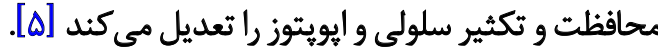

سولفيد هيدروثن التهاب عصبى القاشده با لييويلى ساكاريدها

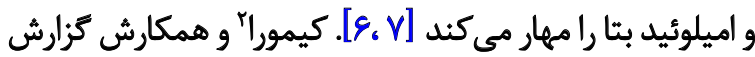

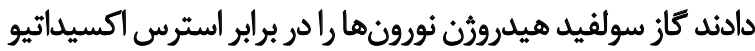

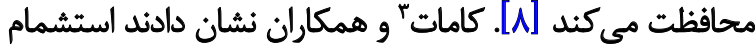

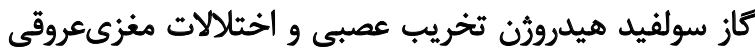

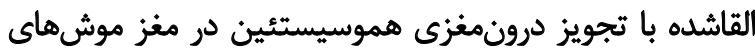

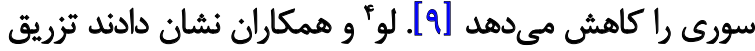

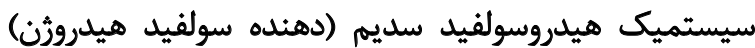
مرى نورونهاى دويامينرزيك بر اثر تجويز سمريد مثيل سيل فنيل

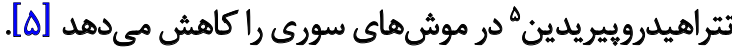
كيداؤ همكاران كزارش كردند استنشاق سولفيد هيدرورن از

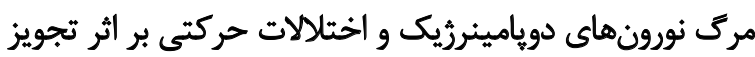

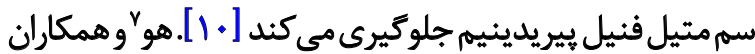

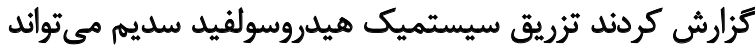

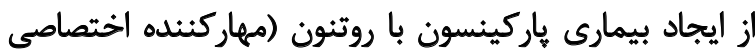

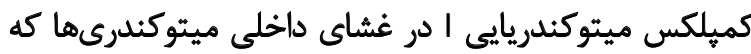

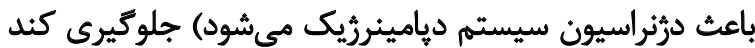

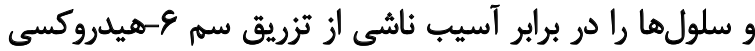

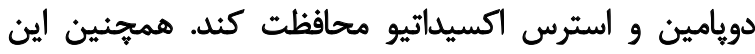

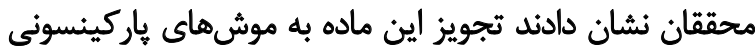

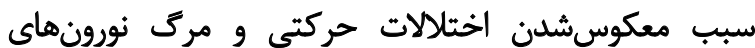

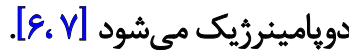

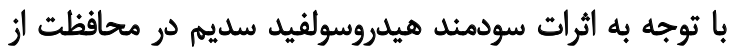

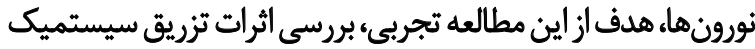

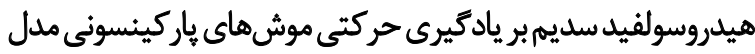

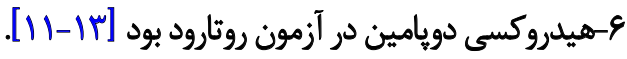

\section{مواد ورشها}

در اين مطالعه \&ه موش صحرايى نر ويستار (1 رأس موش

1. Long Term Potentiation (LTP)

2. Kimura

3. Kamat

4. Lu

5. 1-Methyl-4-Phenyl-1,2,3,6-TetrahydroPyridine (MPTP)

6. Kida

7. $\mathrm{Hu}$ 
اختيلاف بين كروه كنترل و آزمايش با هجهار كروه ياركينسونى و

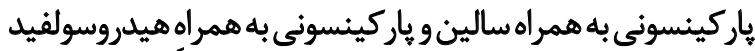

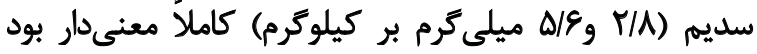

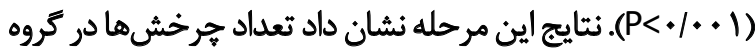

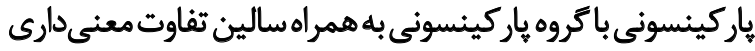

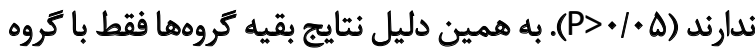

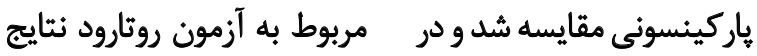

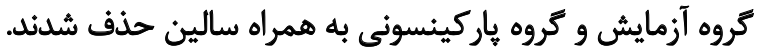

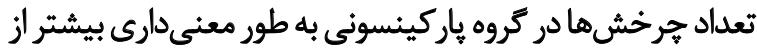

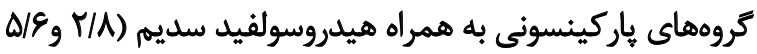

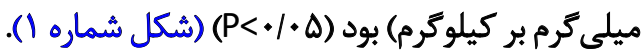

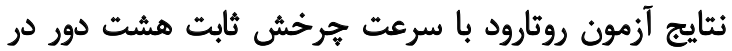

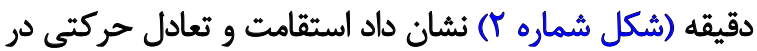

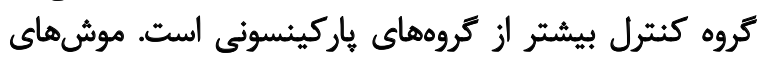

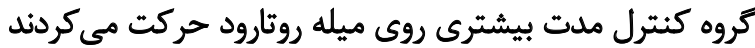

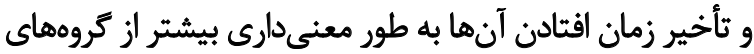

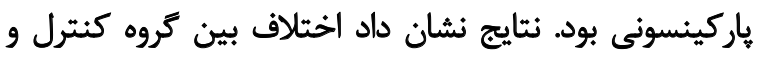

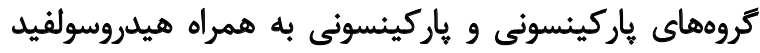

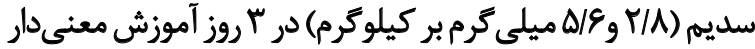

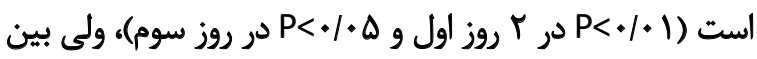

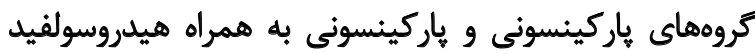

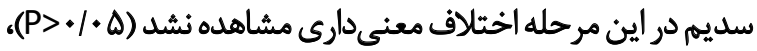

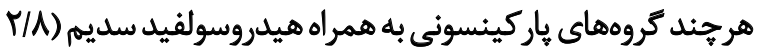

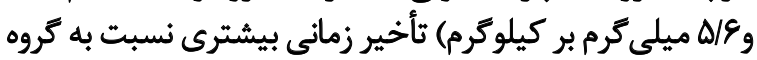

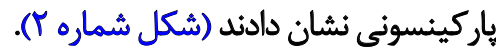

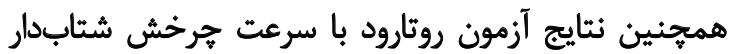

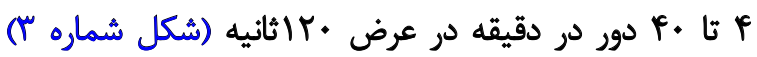

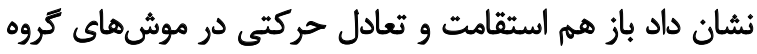

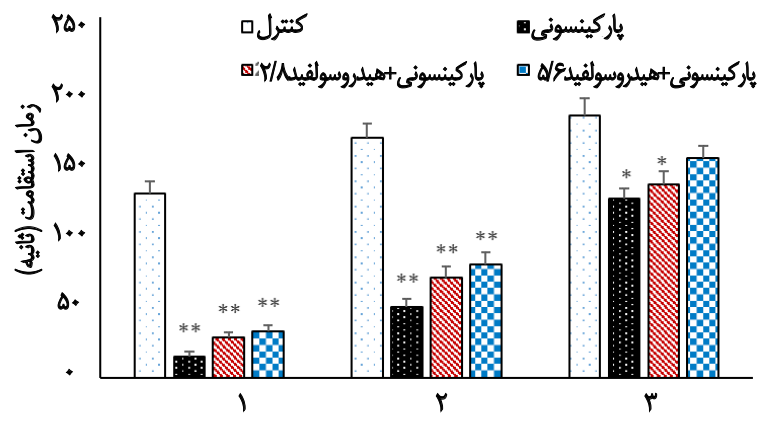

js)

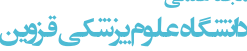

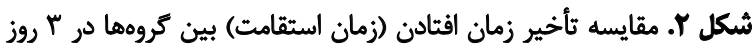

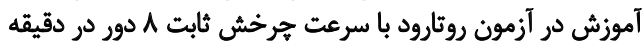

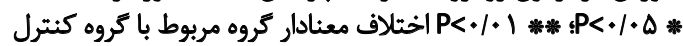

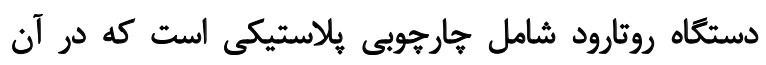

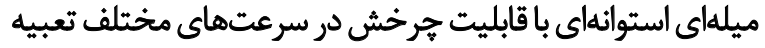

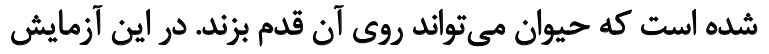

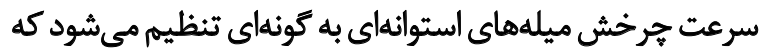

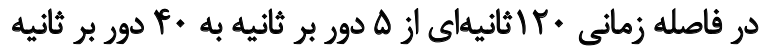

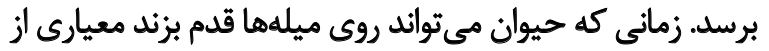
توانايى اجراى حركتى حيوان است

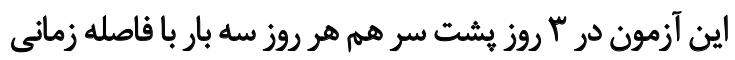

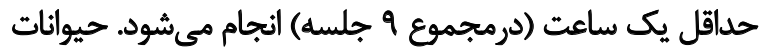

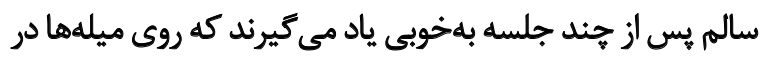

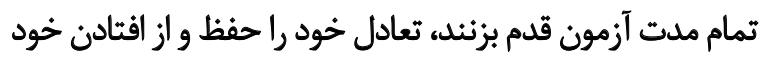

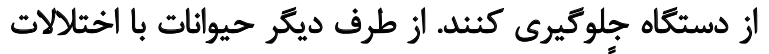

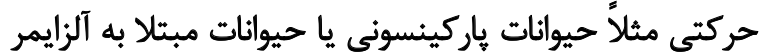

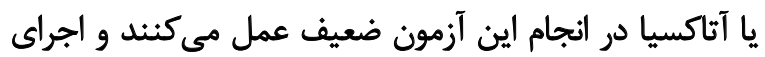

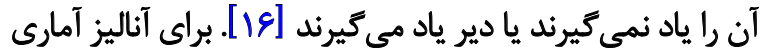

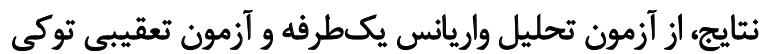

|ستفاده شد.

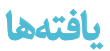

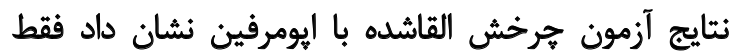

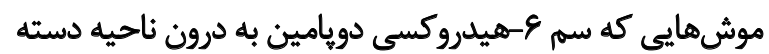

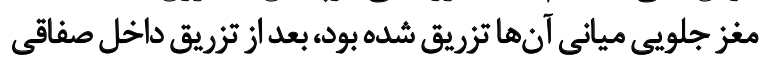

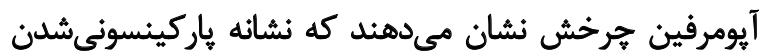

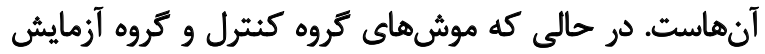

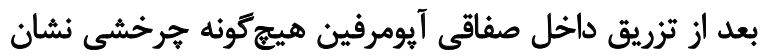

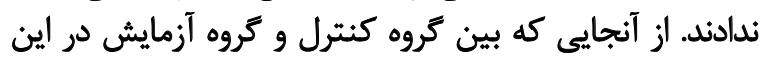

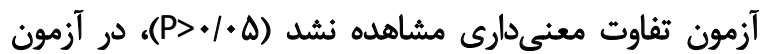
روتارود فقط ازكروه كنترل استفاده شد.

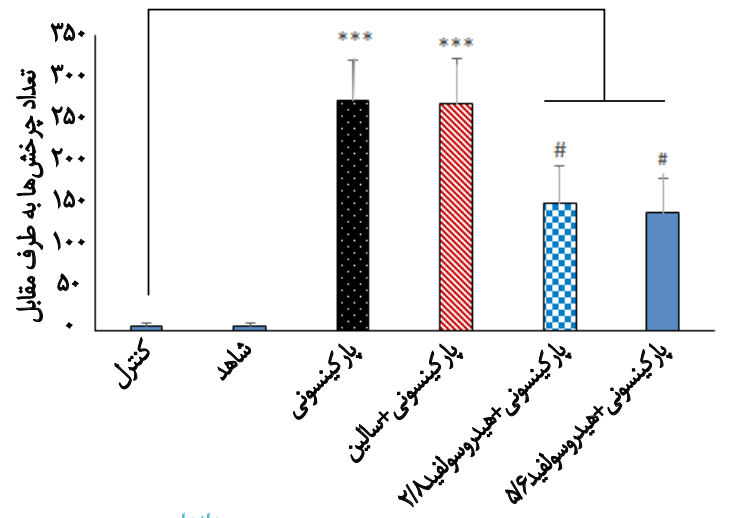

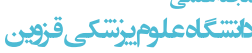

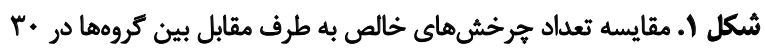

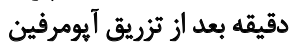

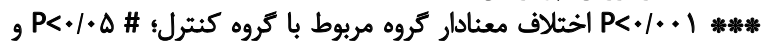

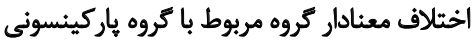


حركتى و اختلالات مغزىعروقى در موشهاى هاركينسونى

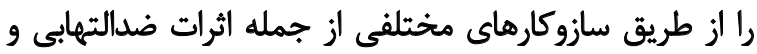

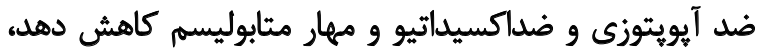

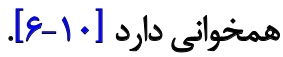
همجنين بانتايجمحققانى كهنشان دادهاندهيدروسولفيدسديم

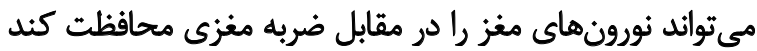

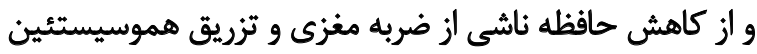

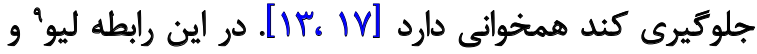

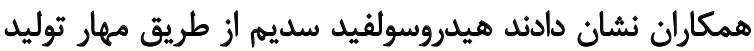

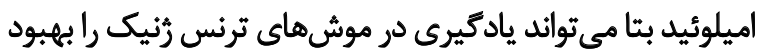

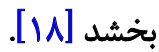
همجنين تجويز دُز كم هيدروسولفيد سديم مى تواند به طور

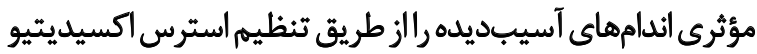

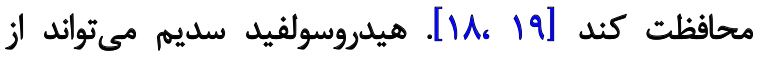

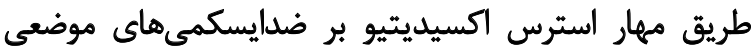

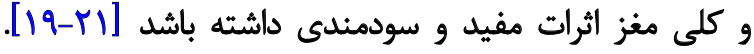

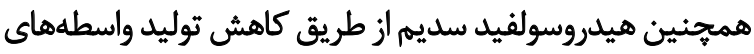

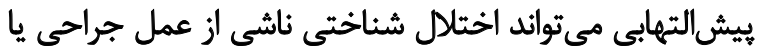

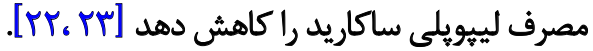
همينطور هيدروسولفيد سديم ميتواند از طريق افزايش

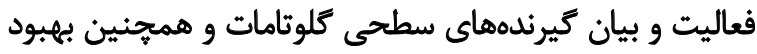

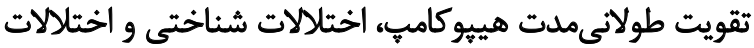

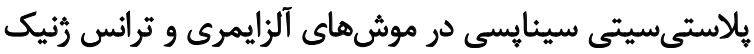

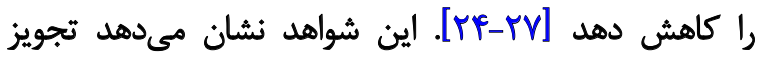

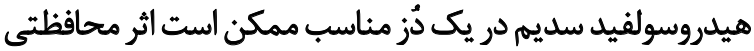

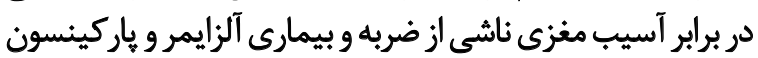

داشته باشد [19].

بيمارى ياركينسون يك اختلال تخريبكنينده عصبى است كه

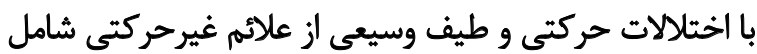

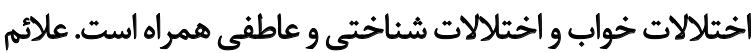

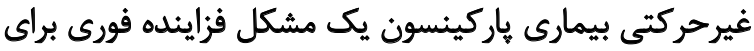

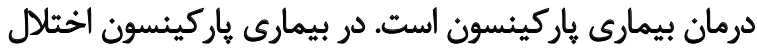

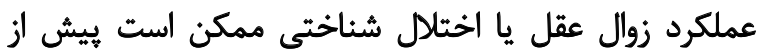

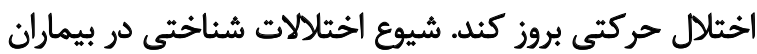

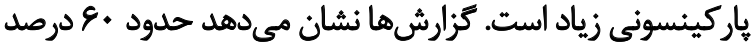

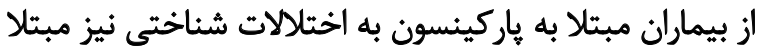

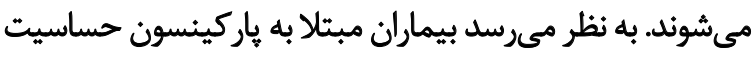

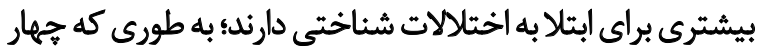

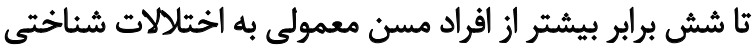

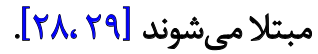

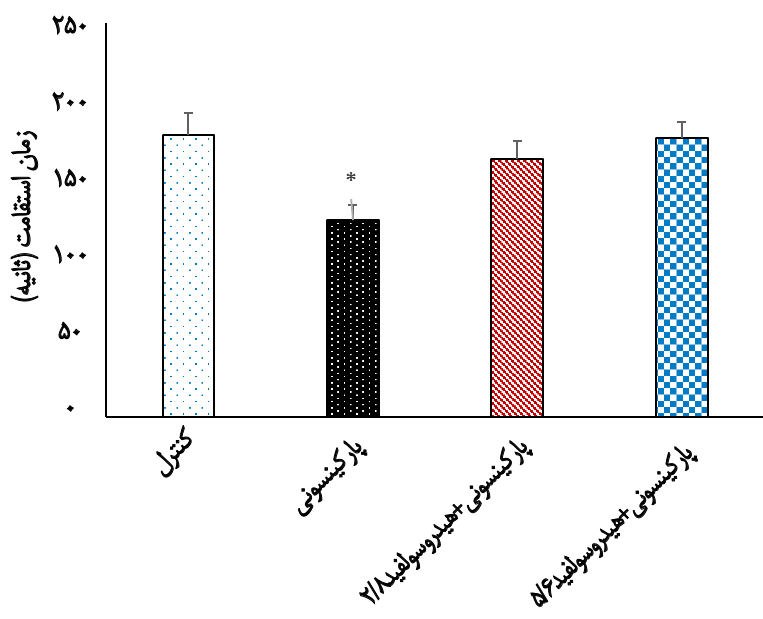

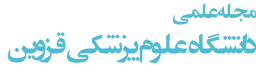

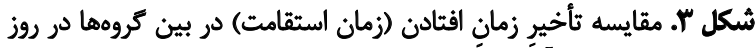

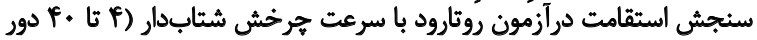

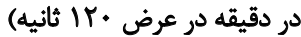
اختلاف معنادار كروه مربوط بانيه مروه كثترل

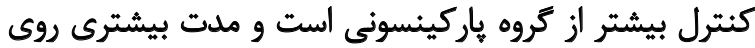

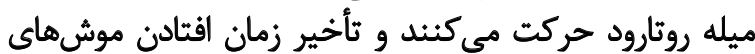

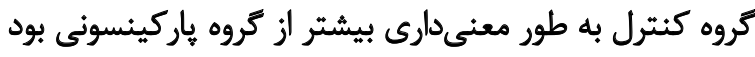

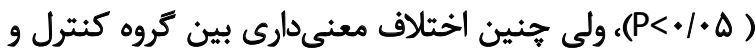

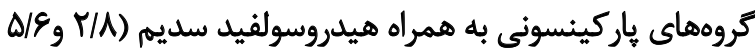

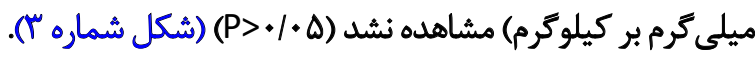

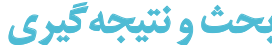

سم \&-هيدروكسى ديامين بسيار نايايدار است و بهآسانى

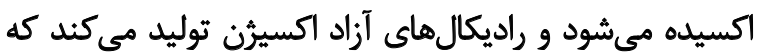

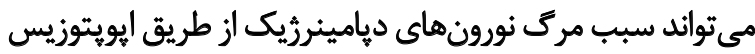

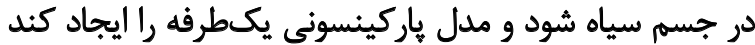

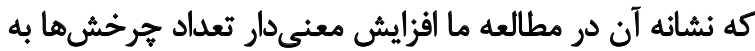

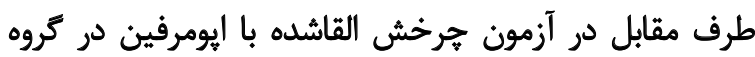

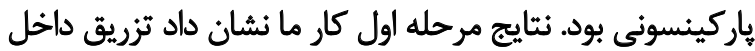

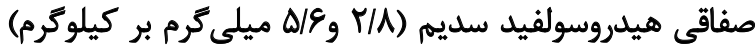

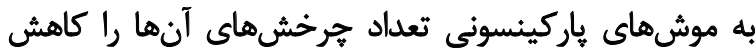

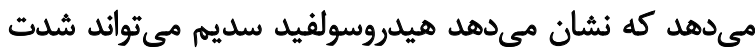

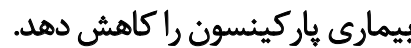

همجنين نتايج مرحله دوم مطالعه حاضر نشان دادئ داد

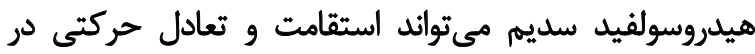

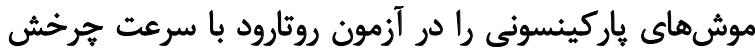

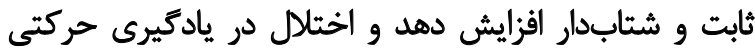

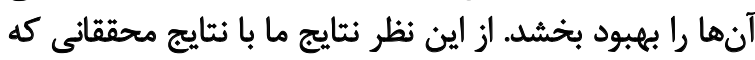

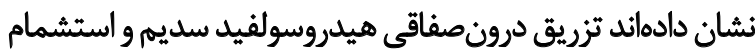

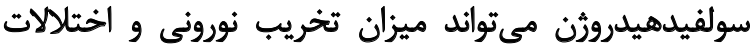




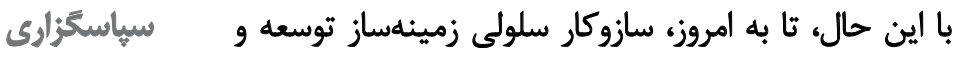

بدين قوسيله از همكارى معاونت ئروهشى دانشكاه علوم يزشكى قزوين تشكر و قدردانى مي ارشود.

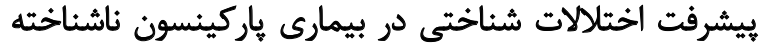

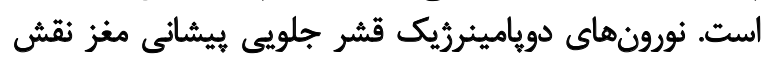

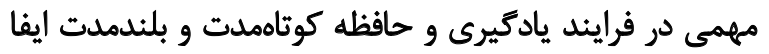

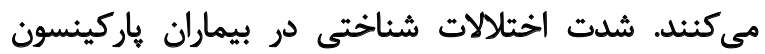

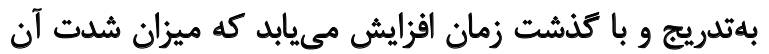

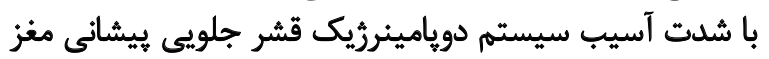

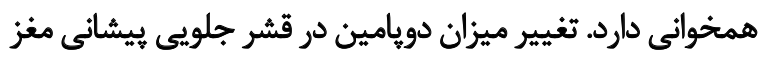

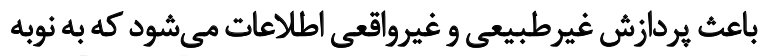

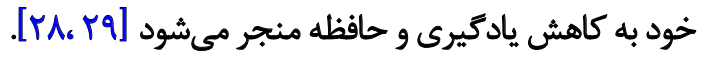
همه اين شواهد نشان مىدهد باركينسونى كردن موشيدها

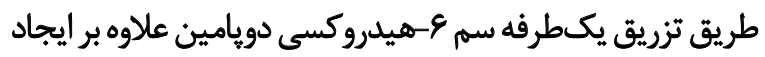

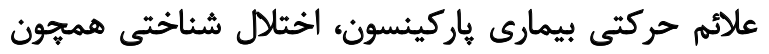

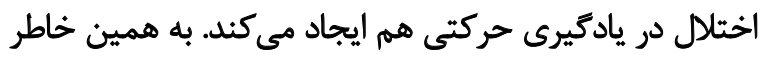

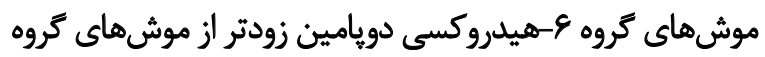

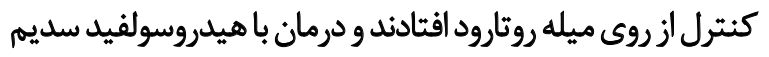

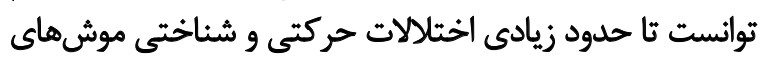

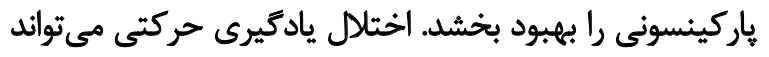

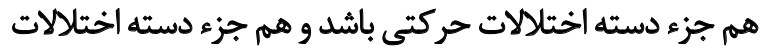

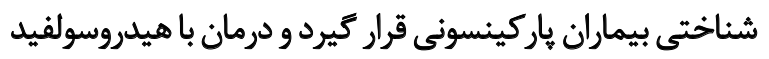
سديم براى آنها سودمند خواهد بودين قرار كيرد

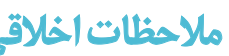

$$
\text { يبيروى از اصول اخلاق يُؤشش }
$$

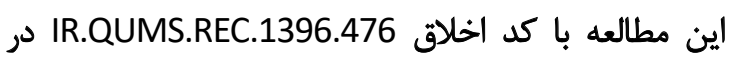
دانشكاه علوم يزشكى قزوين ثبت شده است.

$$
\text { حامي مالّى }
$$

اين مقاله از يايانامه ندا فرشاد، مقطع كارشناسى ارشده در

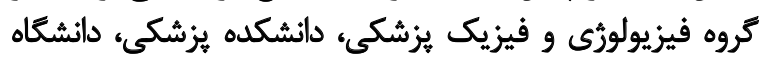
علوم يزشكى قزوين كرفته شده است فئيك

$$
\text { مشاركت نويسند انَّان }
$$

طراح اصلى و بازبينى مقاله به لحاظ محتوايى: محمد حسين

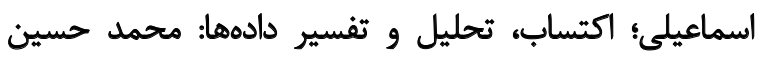

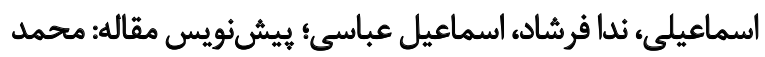
حسين اسماعيلى، هاشم حقدوست يزدئ.

$$
\text { تقارض مناقع }
$$

بنابر اظهار نويسندكانء اين مقاله تعارض منافع ندارد. 


\section{References}

[1] Przedborski S. Pathogenesis of nigral cell death in Parkinson's disease. Parkinsonism Relat Disord. 2005; 11(Suppl1):S3-7. [DOI:10.1016/j.parkreldis.2004.10.012] [PMID]

[2] Dauer W, Przedborski S. Parkinson's disease: Mechanisms and models. Neuron. 2003; 39(6):889-909. [DOI:10.1016/S08966273(03)00568-3]

[3] Tsang AH, Chung KK. Oxidative and nitrosative stress in Parkinson's disease. Biochim Biophys Acta (BBA)-Mol Basis of Dis. 2009; 1792(7):643-50. [DOI:10.1016/j.bbadis.2008.12.006] [PMID]

[4] Wang XJ, Xu JX. Possible involvement of $\mathrm{Ca}^{2+}$ signaling in rotenone-induced apoptosis in human neuroblastoma SH-SY5Y cells. Neurosci Lett. 2005; 376(2):127-32. [DOI:10.1016/j.neulet.2004.11.041] [PMID]

[5] Lu M, Zhao FF, Tang JJ, Su CJ, Fan Y, Ding JH, et al. The neuroprotection of hydrogen sulfide against MPTP-induced dopaminergic neuron degeneration involves uncoupling protein 2 rather than ATP-sensitive potassium channels. Antioxid Redox Signal. 2012; 17(6):849-59. [DOI:10.1089/ars.2011.4507] [PMID] [PMCID]

[6] Hu LF, Lu M, Tiong CX, Dawe GS, Hu G, Bian JS. Neuroprotective effects of Hydrogen Sulfide on Parkinson's Disease rat models. Aging Cell. 2010; 9(2):135-46. [DOI:10.1111/j.14749726.2009.00543.x] [PMID]

[7] Xie L, Hu LF, Teo XQ, Tiong CX, Tazzari V, Sparatore A, et al. Therapeutic effect of Hydrogen Sulfide-releasing L-Dopa derivative ACS84 on 6-OHDA-induced Parkinson's disease rat model. PLoS One. 2013; 8(4):e60200. [DOI:10.1371/journal. pone.0060200] [PMID] [PMCID]

[8] Kimura Y, Kimura H. Hydrogen Sulfide protects neurons from oxidative stress. FASEB J. 2004; 18(10):1165-7. [DOI:10.1096/ fj.04-1815fje] [PMID]

[9] Kamat PK, Kalani A, Givvimani S, Sathnur PB, Tyagi SC, Tyagi $\mathrm{N}$. Hydrogen sulfide attenuates neurodegeneration and neurovascular dysfunction induced by intracerebral-administered homocysteine in mice. Neurosci. 2013; 252:302-19. [DOI:10.1016/j.neuroscience.2013.07.051] [PMID] [PMCID]

[10] Kida K, Yamada M, Tokuda K, Marutani E, Kakinohana M, Kaneki $\mathrm{M}$, et al. Inhaled hydrogen sulfide prevents neurodegeneration and movement disorder in a mouse model of Parkinson's disease. Antioxid Redox Signal. 2011; 15(2):343-52. [DOI:10.1089/ars.2010.3671] [PMID] [PMCID]

[11] Fujita K, Yamafuji M, Nakabeppu Y, Noda M. Therapeutic approach to neurodegenerative diseases by medical gases: Focusing on redox signaling and related antioxidant enzymes. Oxid Med Cell Longev. 2012; 2012:324256. [DOI:10.1155/2012/324256]

[12] Zhou CF, Tang XQ. Hydrogen Sulfide and nervous system regulation. Chinese Med J. 2011; 124(21):3576-82. [PMID]

[13] Karimi SA, Hosseinmardi N, Janahmadi M, Sayyah M, Hajisoltani R. The protective effect of Hydrogen Sulfide $\left(\mathrm{H}_{2} \mathrm{~S}\right)$ on Traumatic Brain Injury (TBI) induced memory deficits in rats.
Brain Res Bull. 2017; 134:177-82. [DOI:10.1016/j.brainresbull.2017.07.014] [PMID]

[14] Nikkhah G, Duan WM, Knappe U, Jödicke A, Björklund A. Restoration of complex sensorimotor behavior and skilled forelimb use by a modified nigral cell suspension transplantation approach in the rat Parkinson model. Neurosci. 1993; 56(1):33-43. [DOI:10.1016/0306-4522(93)90559-X]

[15] Paxinos G, Watson C. The rat brain in stereotaxic coordinates, $3^{\text {rd }}$ ed. San Diego: Academic Press; 1977.

[16] Hamm RJ, Pike BR, O’Dell DM, Lyeth BG, Jenkins LW. The rotarod test: An evaluation of its effectiveness in assessing motor deficits following traumatic brain injury. J Neurotrauma. 1994; 11(2):187-96. [DOI:10.1089/neu.1994.11.187] [PMID]

[17] Li M, Zhang P, Wei HJ, Li MH, Zou W, Li X, et al. Hydrogen sulfide ameliorates homocysteine-induced cognitive dysfunction by inhibition of reactive aldehydes involving upregulation of ALDH2. Int J Neuropsychopharmacol. 2017; 20(4):305-15. [PMID] [DOI: 10.1093/ijnp/pyw103]

[18] Liu Y, Deng Y, Liu H, Yin C, Li X, Gong Q. Hydrogen Sulfide ameliorates learning memory impairment in APP/PS1 transgenic mice: A novel mechanism mediated by the activation of Nrf2. Pharmacol Biochem Behav. 2016; 150-151:207-16. [DOI:10.1016/j.pbb.2016.11.002] [PMID]

[19] Jiang X, Huang Y, Lin W, Gao D, Fei Z. Protective effects of Hydrogen Sulfide in a rat model of traumatic brain injury via activation of mitochondrial adenosine triphosphate-sensitive potassium channels and reduction of oxidative stress. J Surg Res. 2013; 184(2):27-35. [DOI:10.1016/j.jss.2013.03.067] [PMID]

[20] Tan BH, Wong PT, Bian JS. Hydrogen sulfide: A novel signaling molecule in the central nervous system. Neurochem Int. 2010; 56(1):3-10. [DOI:10.1016/j.neuint.2009.08.008] [PMID]

[21] Yin J, Tu C, Zhao J, Ou D, Chen G, Liu Y, et al. Exogenous hy drogen sulfide protects against global cerebral ischemia/reperfusion injury via its anti-oxidative, anti-inflammatory and anti-apoptotic effects in rats. Brain Res. 2013; 1491:188-96. [DOI:10.1016/j.brainres.2012.10.046] [PMID]

[22] Chu QJ, He L, Zhang W, Liu CL, Ai YQ, Zhang Q. Hydrogen sulfide attenuates surgical trauma-induced inflammatory response and cognitive deficits in mice. J Surg Res. 2013; 183(1):330-6. [DOI:10.1016/j.jss.2012.12.003] [PMID]

[23] Gong QH, Wang Q, Pan LL, Liu XH, Huang H, Zhu YZ. Hy drogen sulfide attenuates lipopolysaccharide-induced cognitive impairment: A pro-inflammatory pathway in rats. Pharmac Bioch and Behav. 2010; 96(1):52-8. [DOI:10.1016/j. pbb.2010.04.006] [PMID]

[24] Zhang H, Gao Y, Zhao F, Dai Z, Meng T, Tu S, et al. Hydrogen sulfide reduces mRNA and protein levels of beta-site amyloid precursor protein cleaving enzyme 1 in PC12 cells. Neurochem Int. 2011; 58(2):169-75. [DOI:10.1016/j.neuint.2010.11.010] [PMID]

[25] Xuan A, Long D, Li J, Ji W, Zhang M, Hong L, et al. Hydrogen sulfide attenuates spatial memory impairment and hippocampal neuroinflammation in beta-amyloid rat model of Alzheimer's Disease. J Neuroinflammation. 2012; 9(1):202. [DOI:10.1186/1742-2094-9-202] [PMID] [PMCID] 
[26] He XL, Yan N, Zhang H, Qi YW, Zhu L, Liu MJ, et al. Hydrogen sulfide improves spatial memory impairment and decreases production of $A \beta$ in APP/PS1 transgenic mice. Neurochem Int. 2014; 67:1-8. [DOI:10.1016/j.neuint.2014.01.004] [PMID]

[27] Yang YJ, Zhao Y, Yu B, Xu GG, Wang W, Zhan JQ, et al. GluN2Bcontaining NMDA receptors contribute to the beneficial effects of hydrogen sulfide on cognitive and synaptic plasticity deficits in APP/PS1 transgenic mice. Neurosci. 2016; 335:17083. [DOI:10.1016/j.neuroscience.2016.08.033]

[28] Dallé E, Daniels WM, Mabandla MV. Fluvoxamine maleate effects on dopamine signaling in the prefrontal cortex of stressed Parkinsonian rats: Implications for learning and memory. Brain Res Bull. 2017; 132:75-81. [DOI:10.1016/j.brainresbull.2017.05.014] [PMID]

[29] Masini D, Lopes Aguiar C, Bonito Oliva A, Papadia D, Andersson $\mathrm{R}$, Fisahn $\mathrm{A}$, et al. The histamine $\mathrm{H} 3$ receptor antagonist thioperamide rescues circadian rhythm and memory function in experimental parkinsonism. Transl Psychiatry. 2017; 7(4):e1088. [DOI:10.1038/tp.2017.58] [PMID] [PMCID] 